\title{
Analysis of results of the aggregate microtexture evaluation by volumetric characteristics
}

\author{
Zuzana Florková ${ }^{1,}$, Štefan Šedivý ${ }^{2}$, and Lubomir Pepucha ${ }^{1}$ \\ ${ }^{1}$ University of Zilina, Research Centre, Univerzitna 8215/1, Zilina SK- 010 26, Slovak Republic \\ ${ }^{2}$ University of Zilina, Department of Construction Management, Univerzitna 8215/1, Zilina SK- 010 \\ 26, Slovak Republic
}

\begin{abstract}
The article is concerned about the evaluation of aggregate microtexture. It is very important parameter from the traffic safety point of view and is described as a configuration of small irregularities on the aggregate surface. The new evaluating approach of aggregate microtexture based on the 3-D method is described in this article. 3-D model of surface of aggregate particle was generated from the measurements by microscope method. New program was developed for quantification of aggregate microtexture by a volumetric characteristics. Usability of this method was tested on samples of two coarse aggregate fraction taken from the eleven different quarries in Slovakia. The results of this evaluation have shown a high variability of values of volumetric characteristics between the fractions of aggregate and also between quarries.
\end{abstract}

\section{Introduction}

Microtexture of asphalt pavement is significant surface characteristics in term of skid resistance. It is defined by wavelengths in range $1 \mu \mathrm{m}$ to $0.5 \mathrm{~mm}$ and is characterized by microtexture of particular aggregate used in pavement. Its importance consist in providing basic level of friction between tire rubber and the pavement surface (significant on dry pavement surface by low speed up to $40 \mathrm{kmph}$ ). The effect of microtexture on the pavement skid resistance is in detail described in [1]. From the geometric point of view the microtexture is defined as a configuration of particular peaks on the aggregate surface [2]. It is clear, microtexture depends on the geometric properties of the aggregate grain (shape and size) and also on petrological and physical properties of aggregate grain [3]. Consequently, the type of used aggregate by its properties predetermine the microtexture level of pavement surface.

A lot of different approaches of aggregate microtexture evaluation methods have been developed and a wide range of measurement methods have been developed for its quantifying. However, taking into account their wavelength in micron scale, the possibility to direct accurate quantification is still problematic and the indirect quantification

* Corresponding author: zuzana.florkova@rc.uniza.sk 
techniques with the pendulum tester [4] or dynamic friction tester [5] are used as an alternate.

At the present time, laboratory digital image analysis methods (various complex systems, laser scanner, photogrammetry method or microscopes) are extended for direct quantifying of aggregate microtexture. These methods allows to obtain a digital image of aggregate grain that is obviously transferred into 3-D. A video or camera is used to take photos for 3-D image generation in the complex systems developed for evaluation of aggregate microtexture [6-10]. The use of a laser scanner for 3-D image of aggregate grain and shape properties evaluation is presented in [11].

The main disadvantage of these above mentioned methods is, although 3-D surface models of aggregate grain are available they allow microtexture evaluation of grains only by $2-\mathrm{D}$ characteristics which are mostly based on the shape of aggregate grain. These characteristics are closely presented in [12-15]. In general, disadvantage of 2-D characteristics based on digital image analysis is that these parameters allow to capture only larger peaks on the surface of aggregate grains. As the result of this fact, only the upper part of aggregate microtexture range is included into the evaluation [16]. Information including the evaluation of aggregate surface microtexture using volumetric characteristics derived from the 3-D model of aggregate surface could be found rarely. The example is the evaluation presented in [17]. In this study, the 3-D model of aggregate grain resulting from the photogrammetry method was used. The peak material volume characteristics (Vmp) was determined from 3-D model surface based on the Abbott-Firestone curve and only top parts of aggregate grains were included into the evaluation of the microtexture.

Lack of knowledge has been a motive for the research in this field. The main purpose was to use the 3-D model of aggregate surface obtained by optical microscopy as a basic evaluation input and find an adequate volumetric characteristics for following evaluation.

\section{Principle of measurement by microscope method}

Optical microscope NIKON AZ 100 (Fig.1) was used to obtain 3-D model of aggregate grains with different surface microtexture. The principle of this measurement rest in scanning of investigated particle by cameras at different Z-axis planes.
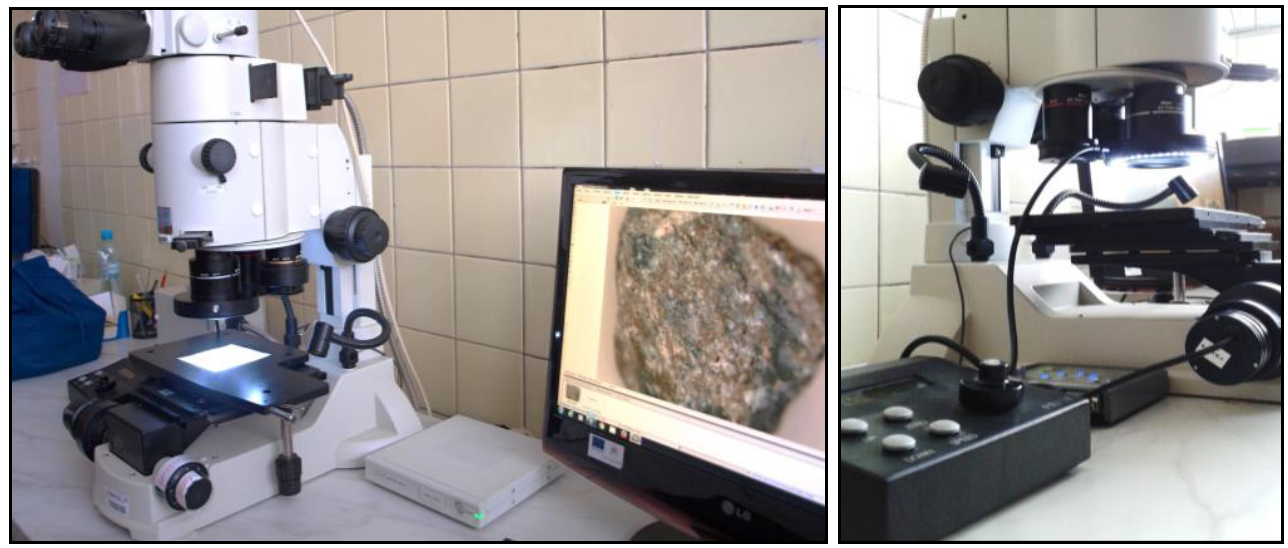

Fig. 1. Optical microscope NIKON AZ 100.

The investigated particle is set on the microscope pad. This pad is during scanning vertically moved in $\mathrm{Z}$-axis direction (in micro steps). After scanning process, the microscope imaging software NIS Elements Documentation enables tag together images that have been scanned in a different $\mathrm{Z}$-axis planes and then creating a resultant 3-D model. 
This 3-D model of investigated particle can be exported into the Virtual Reality Modelling Language (Wrml) format (matrix form of regular placed height points of investigated grain). It allows to display and process the obtained 3-D model also apart from the appropriate microscope software in the working background of different computing programs. Then, a choices computing program can be used for following evaluation.

\section{Evaluation of aggregate microtexture}

\subsection{Determination of volumetric characteristics}

The new evaluation method was developed in the Matlab computing program with the aim of quantification microtexture of aggregate grain. This method uses as a basic input 3-D model in Wrml format, determined by microscope measurement. The main principle of this method is in definition of volume between particular peaks on the aggregate surface. Basically, it is a volume between "real" and convex surface of aggregate grain (Fig. 2). Then, this volume presented microtexture of aggregate grain and is determined by characteristics $\mathrm{Zvd}$ ( $Z$ - plane volume difference). It is a new volumetric microtexture evaluating characteristics which determined aggregate microtexture value by percentage difference of two volumes.
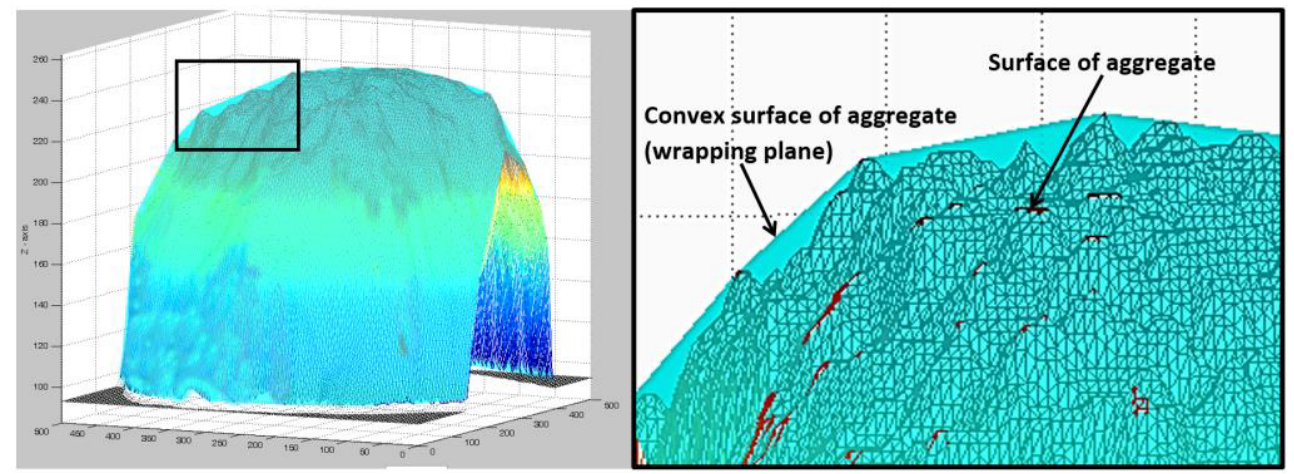

Fig. 2. 3-D model of aggregate particle.

The particular volumes are calculated from the defined comparative plane. First volume is determined as the volume of the aggregate grain and the second volume is determined as the volume of wrap (volume under the wrapping plane). Then, smaller value of Zvd characteristics defines lower suitable microtexture and vice versa. That higher value of Zvd characteristics defines angular aggregate with more peaks on the aggregate surface in comparison to polished or rounded aggregate. For more simply and practically calculation of Zvd characteristics was developed the program MicoSYS. This program allows evaluation of aggregate microtexture of only a selected part of aggregate particle by options of height adjustment of comparative (cutting) plane in the Z-axis direction. The principle and determination of $\mathrm{Zvd}$ characteristics and program MicroSyS are in detail described in [18].

\subsection{Measurement and evaluation conditions}

Usability of this evaluation method was tested on aggregate samples taken from eleven different quarries in Slovakia. The aggregates produced from these quarries fulfill all requirements from aspect of road construction quality. The aggregates differed from 
petrography point of view and ranged from soft (dolomite) to hard (andesite). Two fractions of aggregate particles (4/8 and 8/11) taken from each quarry were used in investigation. These fractions are the most important to ensure the adequate level of microtexture of pavement wearing course. Each sample of the quarry consists of ten aggregate grains (five grains from each fraction). Particular grains were scanned by the stereomicroscope. The measurement process was the same in the case of all scanning aggregates and was performed with the total magnification of 12.5 for the purpose of capturing the largest surface area of scanned aggregate. This magnification represents accuracy $57 \mu \mathrm{m}$ in X-axis and $\mathrm{Y}$-axis direction (in relation to dimensions of scanned area). The scanning range was divide into 400 steps. It represents $10-30 \mu \mathrm{m}$ accuracy in Z-axis direction (in relation to aggregate height). Thereafter, the MicroSYS program was used to calculate value of microtexture volumetric characteristics $\mathrm{Zvd}$ for each tested aggregate grain. The position of the comparative plane was chosen in the same position for all aggregate grains to have comparable results.

\section{Analysis of results}

The representative value of aggregate microtexture for each quarry and fraction was obtained as a mean value from five values of volumetric characteristics Zvd determined on particular aggregate grains. The results of evaluation are presented in Figure 3.

It was supposed the different nature of aggregate in the individual quarries (and various portion of soft and hard minerals in these rocks) in combination with equipment used for aggregate production could influence number, shape and evenness of fracture planes and thus differences of microtexture values among the particular kinds of aggregates will be observed. However, when two fractions are compared, a production equipment should not play any role (obviously the same is used for production both fractions) and a microtexture of aggregate surface should only depend on properties of rock.

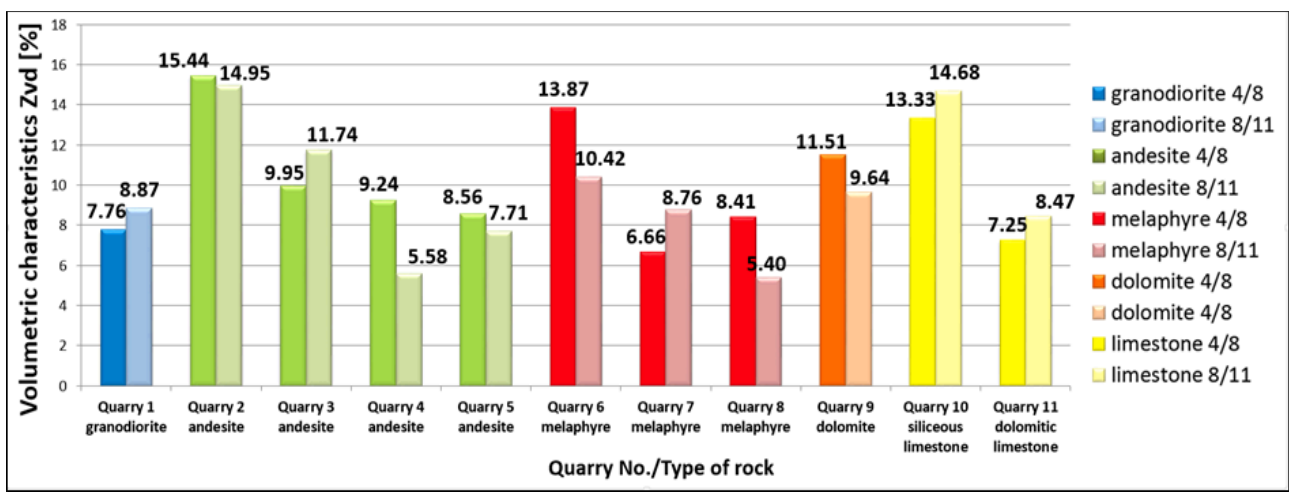

Fig. 3. Resultant values of volumetric characteristics Zvd - fraction 4/8 and 8/11.

It is obviously in the Figure 3, that obtained results have shown the considerable variability. There are the differences between fractions of aggregates and also between the quarries. Therefore, it is not possible expressly to type of particulars types of rocks by the values of determined volumetric characteristics Zvd from the microtexture point of view.

Table 1, summarizes a values variability of volumetric charasteristics Zvd in case of all investigated samples of aggregates form particular quarries and fractions. Data in Table 1 are presented by maximum, minimum and mean values of Zvd charcteristics. For better interpretation, these values are also presented in Figure 4. On the basis of results presented in Table. 1 and Figure 4, it can be argue, that the variability of results obtained by this 
method is very high. In expression as a percentage difference between maximally and minimally obtained values it can achieve for particular sample even $296 \%$.

Table 1. Variability summarization of volumetric characteristics Zvd determined on particular samples of aggregates.

\begin{tabular}{|c|c|c|c|c|c|c|c|c|}
\hline Zvd [\%] & \multicolumn{5}{|c|}{ Fraction 4/8 } & \multicolumn{3}{c|}{ Fraction 8/11 } \\
\hline $\begin{array}{c}\text { Quarry } \\
\text { No. }\end{array}$ & $\begin{array}{c}\text { Mean } \\
\text { Zvd }\end{array}$ & $\begin{array}{c}\text { Min. } \\
\text { Zvd }\end{array}$ & $\begin{array}{c}\text { Max. } \\
\text { Zvd }\end{array}$ & $\begin{array}{c}\text { Percentage } \\
\text { difference } \\
{[\%]}\end{array}$ & $\begin{array}{c}\text { Mean } \\
\text { Zvd }\end{array}$ & $\begin{array}{c}\text { Min. } \\
\text { Zvd }\end{array}$ & $\begin{array}{c}\text { Max. } \\
\text { Zvd }\end{array}$ & $\begin{array}{c}\text { Percentage } \\
\text { difference } \\
{[\%]}\end{array}$ \\
\hline 1 & 7.76 & 5.53 & 8.62 & 55.88 & 8.87 & 5.41 & 12.63 & 133.46 \\
\hline 2 & 15.44 & 10.00 & 25.49 & 154.90 & 14.95 & 7.02 & 22.50 & 220.51 \\
\hline 3 & 9.95 & 6.02 & 15.42 & 156.15 & 11.74 & 6.06 & 22.37 & 269.14 \\
\hline 4 & 9.24 & 6.64 & 13.29 & 100.15 & 5.58 & 4.19 & 7.59 & 81.15 \\
\hline 5 & 8.56 & 6.41 & 11.18 & 74.41 & 7.71 & 5.08 & 11.07 & 117.91 \\
\hline 6 & 13.87 & 7.80 & 23.80 & 205.13 & 10.42 & 5.97 & 23.64 & 295.98 \\
\hline 7 & 6.66 & 4.52 & 8.43 & 86.50 & 8.76 & 6.15 & 10.32 & 67.80 \\
\hline 8 & 8.41 & 5.61 & 15.69 & 179.68 & 5.40 & 2.98 & 8.37 & 180.87 \\
\hline 9 & 11.51 & 6.01 & 21.11 & 251.25 & 9.64 & 5.96 & 11.88 & 99.33 \\
\hline 10 & 13.33 & 9.57 & 22.16 & 131.56 & 14.68 & 10.0 & 19.82 & 96.43 \\
\hline 11 & 7.25 & 5.30 & 10.31 & 94.53 & 8.47 & 6.19 & 11.52 & 86.11 \\
\hline
\end{tabular}

Possible causes can be several. Firstly, it can relates with a low number of grains representing particular aggregate samples. It confirm also differences among values of characteristics Zvd determined on the particular grains of aggregates within investigated samples (Figure 5). By reason of lucidity, the Figure 5 shows only some of quarry samples but it is sufficient for illustration of values difference within investigated grains.

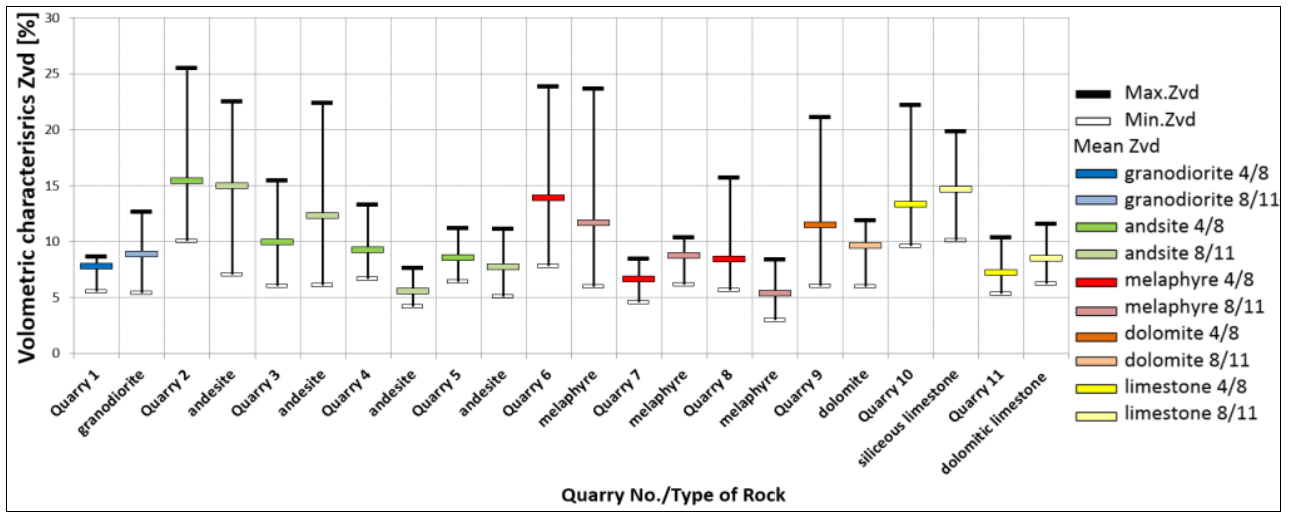

Fig. 4. Values variability of volumetric characteristics Zvd determined on particular samples of aggregates.

Another reason can be probably related with grain size of investigated aggregates. Inspite of equal measurement and evaluation conditions by measurements with the total magnification of 12.5 each aggregate grain has different size and thus always different surface area (below which is calculated volume) enters in the resultant evaluation. It causes 
that basic input entering in the resultant evaluation is always different especially when fractions $4 / 8$ and $8 / 11$ are compared.

It could be influenced also by measurement errors (different reflection or variance light conditions) because of optical microscope method. The insufficient magnification mainly influence the resultant values of characteristics Zvd. It causes that the scanning area of aggregate surface is always different and it affects also the accuracy of obtained microscope output in Wrml format. This format is a standard file format for representing 3-D information and in this case is represented by matrix form of regular placed height points of scanned particle. The mathematic matrix dimensions are 200 points in $\mathrm{X}$-axis direction and 150 points in $\mathrm{Y}$-axis direction. It leads to the lack of microtexture informations by usage a low microscope scanning magnification. On the basis of results obtained so far it can be concluded that the technique of microscope scanning was choose incorrect.

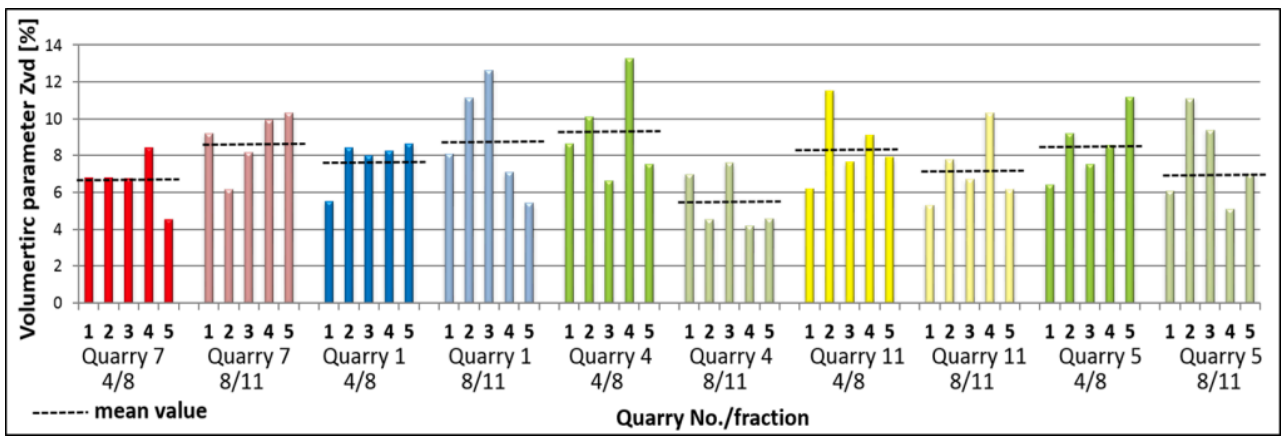

Fig. 5. Values variability of characteristics Zvd determined on the particular grains of aggregates within investigated samples.

\section{Conclusions}

The new approach for microtexture quantification and subsequent evaluation on the basis of 3-D outputs obtained by optical microscope was presented in this paper. Presented method uses new program MicroSYS for calculation of characteristics Zvd ( $Z$ - plane volume difference) as a volumetric characterization of aggregate surface microtexture. Zvd characteristics determined aggregate microtexture value by percentage difference of two volumes - the volume of the aggregate grain and the volume of wrap (volume under the wrapping plane). Applicability of this evaluation method was tested on aggregate samples taken from eleven different quarries in Slovakia. The aggregates differed from petrography point of view and ranged from soft to hard. Two fractions of aggregate particles (4/8 and 8/11) taken from each quarry were used in investigation.

The presented results have shown, that evaluation by characteristics Zvd incommensurate expected results because of considerable variability. A wide differences between fractions of aggregates and between the quarries was found. Therefore, it is possible to argue that it is not possible expressly to type of particulars types of rocks by the values of determined volumetric characteristics Zvd from the microtexture point of view. The results analysis have shown that the main reason of uncertain results relates with incorrect choosing of technique of microscope scanning. For the purpose of confirmation of usability this characteristics Zvd in microtexture evaluation process next test have to be carried out. It is necessary take measurements with higher magnification because the accuracy of the obtained output depends on the size of scanning area thus on the required magnification. With increasing of magnification is decreasing the size of scanning area and also is decreasing the depth of focus. It leads to higher accuracy of scanning resolution and 
also to the constant scanning area of aggregate surface for the all investigates grains. In this case is needed to practise a new measurement.

It is also necessary to carry out measurements on a numerous aggregate grains, which have a different level of microtexture (e.g. granodiorite versus limestone) in order to obtain sufficiently representative microtexture value of investigated aggregate by this method and after confirmation that this method makes it possible to distinguish the aggregate surface to carry out additional measurements.

This research was supported by European project Research Centre of the University of Žilina Second Phase: ITMS 313011D011.

\section{References}

1. J.W. Hall et al, Guide for Pavement Friction. NCHRP Web-Only Document 108, February (2009)

2. CEN. EN ISO 13473-5, Characterization of pavement texture by use of surface profiles, Part 5: Determination of megatexture (2009)

3. K. Scharnigg, G. Schwalbe, TYROSAFE - Report on knowledge gaps and proposals for further research concerning optimisation for road surfaces and tyres for skid resistance rolling resistance and noise emissions (2010)

4. STN EN 13036-4, Road and airfield surface characteristics, Test methods, Part 4: Method for measurement of slip/skid resistance of a surface: The pendulum test (2012)

5. Q. Lu, B. Steven, Friction Testing of Pavement Preservation Treatments: Literature Review (2006)

6. N. H. Maerz, W. Zhou. Flat and Elongated, Advances Using Digital Image Analysis, In: Proc. of the 9th Annual Symposium of the International Centre for Aggregates Research (ICAR) (2001)

7. E. Tutumluer, C. Rao, J. Stefanski, Video Image Analysis of Aggregates, In: Transportation Engineering Series, 111, Illinois Cooperative Highway and Transportation Series (2000)

8. C. Rao, Development of 3-D Image Analysis Techniques to Determine Shape and Size Properties of Coarse Aggregate, PhD. Thesis, Department of Civil Engineering, University of Illinois at Urbana-Champaign (2001)

9. E. Masad, The Development of a Computer Controlled Image Analysis System for Measuring Aggregate Shape Properties, National Cooperative Highway Research Program NCHRP-IDEA Project 77, Final report, (2003)

10. H. Kim et al, A Prototype Laser Scanner for Characterizing Size and Shape Properties in Aggregates, In: Proc. of the 9th Annual Symposium of the International Centre for Aggregate Research (ICAR) (2001)

11. J.K. Anochie-Boateng et al, Evaluation of 3D laser device for characterizing shape and surface properties of aggregates used in pavements, In: 29th Southern Africa Transportation Conference (2010)

12. E. Masad et al, Test Methods for Characterizing Aggregate Shape Texture and Angularity, Final report - appendixes, National Cooperative Highway Research Program, Appendixes to NCHRP Report 555 (2005)

13. Z. Florková, Microtexture of coarse aggregate for asphalt pavements, Diploma thesis, University of Žilina, Faculty of Civil Engineering, Department of Highway Engineering (In Slovak) (2012) 
14. S. Tafesse et al, Sedimentology, 60, 4, 1100-1110 (2013)

15. T. Al-Rousan et al., Cons. and Build. Mat, 21, 5, 978-990 (2007)

16. Z. Florková, Communications, 17, 4 (2015) p. 70-74.

17. G. Mcquaid et al., Intern. J. of Pavements Conference, Sao Paulo, (2013)

18. Z. Florková, Microtexture evaluation of asphalt pavement surface, PhD. Thesis, University of Žilina, Faculty of Civil Engineering, Department of Highway Engineering, (In Slovak) (2016) 